\title{
REMARK ON THE INTEGRALS OF MOTION ASSOCIATED WITH LEVEL $k$ REALIZATION OF THE ELLIPTIC ALGEBRA U $q, p\left(\widehat{s i}_{2}\right)$
}

\author{
TAKEO KOJIMA AND JUN'ICHI SHIRAISHI
}

Communicated by Gaetano Vilasi

Abstract. We give one parameter deformation of level $k$ free field realization of the screening current of the elliptic algebra $\mathrm{U}_{q, p}\left(\widehat{\mathfrak{s l}_{2}}\right)$. By means of these free field realizations, we construct infinitely many commutative operators, which are called the nonlocal integrals of motion associated with the elliptic algebra $\mathrm{U}_{q, p}\left(\widehat{\mathfrak{s l}_{2}}\right)$ for level $k$. They are given as integrals involving a product of the screening current and elliptic theta functions. This paper give level $k$ generalization of the nonlocal integrals of motion given in [1].

\section{Introduction}

One of the results in Bazhanov, Lukyanov and Zamolodchikov [4] is construction of field theoretical analogue of the commuting transfer matrix $\mathbf{T}(z)$, acting on the highest weight representation of the Virasoro algebra. Their commuting transfer matrix $\mathbf{T}(z)$ is the trace of the image of the universal $R$-matrix associated with the quantum affine symmetry $\mathrm{U}_{q}\left(\widehat{\mathfrak{s l}_{2}}\right)$. This construction is very simple and the commutativity $[\mathbf{T}(z), \mathbf{T}(w)]=0$ is direct consequence of the Yang-Baxter equation. They call the coefficients of the Taylor expansion of $\mathbf{T}(z)$ the nonlocal integrals of motion. The higher-rank generalization of [4] is considered in $[5,6]$. The elliptic deformation of the nonlocal integrals of motion is considered in [1]. Bazhanov, Lukyanov and Zamolodchikov [4] constructed the continuous transfer matrix $\mathbf{T}(z)$ by taking the trace of the image of the universal $R$-matrix associated with $\mathrm{U}_{q}\left(\widehat{\mathfrak{s l}_{2}}\right)$. However, it is not so easy to calculate the image of the elliptic version of the universal $R$-matrix, which is obtained by using the twister [10]. Hence the construction method of the elliptic version [1] should be completely different from those in [4]. Instead of considering the transfer matrix $\mathbf{T}(z)$, the authors [1] give the integral representation of the integrals of motion directly. The commutativity of the integrals of motion is not consequence of the Yang-Baxter equation. It is consequence of the commutative subalgebra of the Feigin-Odesskii 
algebra [11]. The higher-rank generalization of [1] is considered in [2,3]. This paper is a continuation of [1-3]. This paper give level $k$ generalization of the nonlocal integrals of motion given in [1].

The organization of this paper is as following. In Section 2 we give one parameter " $s$ " deformation of the level $k$ free field realization of the screening current of the elliptic algebra $\mathrm{U}_{q, p}\left(\widehat{\mathfrak{s l}_{2}}\right)$. In Section 3 we construct infinitely many commutative operators, which are called the nonlocal integrals of motion associated with the elliptic algebra $\mathrm{U}_{q, p}\left(\widehat{\mathfrak{s l}_{2}}\right)$ for level $k$. In Section 3 we state main theorem and give conjecture. In appendix we summarize the normal ordering of basic operators.

\section{Elliptic Current}

In this section we give one parameter " $s$ " deformation of the level $k$ free field realization of the elliptic algebra $\mathrm{U}_{q, p}\left(\widehat{\mathfrak{s l}_{2}}\right)$. We fix complex numbers $x, r, r^{*}, s$, $\left(|x|<1, \operatorname{Re}(r), \operatorname{Re}\left(r^{*}\right)>0, s \neq 2\right)$, and $k=r-r^{*} \neq 0,-2$. We use symbols

$$
[n]=\frac{x^{n}-x^{-n}}{x-x^{-1}}, \quad[n]_{+}=x^{n}+x^{-n} .
$$

We set the parameters $\tau, \tau^{*}$

$$
x=\mathrm{e}^{-\pi \sqrt{-1} / r \tau}=\mathrm{e}^{-\pi \sqrt{-1} / r^{*} \tau^{*}} .
$$

Let us use parameterization $z=x^{2 u}$. The symbol $[u]_{r}$ stands for the Jacobi elliptic theta function

$$
[u]_{r}=x^{\frac{u^{2}}{r}-u} \Theta_{x^{2 r}}(z), \quad[u]_{r^{*}}=x^{\frac{u^{2}}{r^{*}}-u} \Theta_{x^{2 r^{*}}}(z)
$$

where we have used

$$
\Theta_{p}(z)=(z ; p)_{\infty}(p / z ; p)_{\infty}(p ; p)_{\infty}, \quad(z ; p)_{\infty}=\prod_{n=0}^{\infty}\left(1-p^{n} z\right) .
$$

The theta function $[u]_{r}$ enjoys the quasi-periodicity property

$$
[u+r]_{r}=-[u]_{r}, \quad[u+r \tau]_{r}=-e^{-\pi \sqrt{-1} \tau-\frac{2 \pi \sqrt{-1}}{r} u}[u]_{r} .
$$

\subsection{Bosons}

We set the bosons $\alpha_{m}^{j}, \widetilde{\alpha}_{m}^{j} j=1,2, m \in \mathbb{Z}_{\neq 0}$

$$
\left[\alpha_{m}^{j}, \alpha_{n}^{j}\right]=-\frac{1}{m} \frac{[2 m][r m]}{[k m][(r-k) m]} \delta_{m+n, 0}, \quad j=1,2
$$




$$
\begin{aligned}
{\left[\alpha_{m}^{1}, \alpha_{n}^{2}\right]=} & \frac{1}{m}\left(\frac{x^{(-r+k) m}([s m]-[(s-2) m])}{[(r-k) m]}+\frac{x^{k m}([s m]+[(s-2) m])}{[k m]}\right) \\
& \times \delta_{m+n, 0} \\
{\left[\widetilde{\alpha}_{m}^{j}, \widetilde{\alpha}_{n}^{j}\right]=} & -\frac{1}{m} \frac{[2 m][(r-k) m]}{[k m][r m]} \delta_{m+n, 0}, \quad j=1,2 \\
{\left[\widetilde{\alpha}_{m}^{1}, \widetilde{\alpha}_{n}^{2}\right]=} & \frac{1}{m}\left(\frac{x^{r m}(-[s m]+[(s-2) m])}{[r m]}+\frac{x^{k m}([s m]+[(s-2) m])}{[k m]}\right) \\
& \left.\times \delta_{m+n, 0}^{j}, \widetilde{\alpha}_{n}^{j}\right]= \\
{\left[\alpha_{m}^{1}, \widetilde{\alpha}_{n}^{2}\right]=} & \frac{1}{m} \frac{1}{m} \frac{[2 m]}{[k m]} \delta_{m+n, 0}, \quad j=1,2 \\
{\left[\widetilde{\alpha}_{m}^{1}, \alpha_{n}^{2}\right]=} & \frac{1}{m} \frac{[s m]+[(s-2) m]}{[k m]} \delta_{m+n, 0}
\end{aligned}
$$

We set the bosons $\beta_{m}^{j}, \gamma_{m}^{j}, j=1,2, m \in \mathbb{Z}_{\neq 0}$

$$
\begin{aligned}
{\left[\beta_{m}^{j}, \beta_{n}^{j}\right] } & =\frac{[2 m][(k+2) m]}{m} \delta_{m+n, 0}, \quad j=1,2 \\
{\left[\beta_{m}^{1}, \beta_{n}^{2}\right] } & =-\frac{[(k+2) m]([s m]+[(s-2) m])}{m} \delta_{m+n, 0} \\
{\left[\gamma_{m}^{j}, \gamma_{n}^{j}\right] } & =\frac{1}{m} \frac{[2 m]}{[k m]} \delta_{m+n, 0}, \quad j=1,2 \\
{\left[\gamma_{m}^{1}, \gamma_{n}^{2}\right] } & =-\frac{1}{m} \frac{[s m]+[(s-2) m]}{[k m]} \delta_{m+n, 0} .
\end{aligned}
$$

We set the zero-mode operators $P_{0}, Q_{0}, h, \alpha$ and $h_{0}, h_{1}, h_{2}, \alpha_{0}, \alpha_{1}, \alpha_{2}$

$$
\begin{aligned}
{\left[P_{0}, \mathrm{i} Q_{0}\right] } & =1, \quad[h, \alpha]=2 \\
{\left[h_{0}, \alpha_{0}\right] } & =\left[h_{1}, \alpha_{2}\right]=\left[h_{2}, \alpha_{1}\right]=(2-s), \quad\left[h_{1}, \alpha_{1}\right]=\left[h_{2}, \alpha_{2}\right]=0 .
\end{aligned}
$$

We set the Fock space $\mathcal{F}_{K, L}, K, L \in \mathbb{Z}$

$$
\mathcal{F}_{K, L}=\bigoplus_{n, n_{0}, n_{1}, n_{2} \in \mathbb{Z}} \mathbb{C}\left[\alpha_{-m}^{j}, \widetilde{\alpha}_{-m}^{j}, \beta_{-m}^{j}, \gamma_{-m}^{j},\left(j=1,2 ; m \in \mathbb{N}_{\neq 0}\right)\right]
$$

$\otimes|K, L\rangle_{n, n_{0}, n_{1}, n_{2}}$ 
where

$$
|K, L\rangle_{n, n_{0}, n_{1}, n_{2}}=\exp \left(L \sqrt{\frac{2 r}{r-k}}-K \sqrt{\frac{2(r-k)}{r}}\right) \mathrm{i} Q
$$

$\otimes \mathrm{e}^{n \alpha} \otimes \mathrm{e}^{n_{0} \alpha_{0}} \otimes \mathrm{e}^{n_{1} \alpha_{1}} \otimes \mathrm{e}^{n_{2} \alpha_{2}}$.

Upon specialization $s \rightarrow 2$ simplification occurs

$$
\begin{array}{cc}
\alpha_{m}^{2}=-\alpha_{m}^{1}, \quad & \widetilde{\alpha}_{m}^{1}=\frac{[(r-k) m]}{[r m]} \alpha_{m}^{1} \quad \widetilde{\alpha}_{m}^{2}=-\frac{[(r-k) m]}{[r m]} \alpha_{m}^{1} \\
\beta_{m}^{2}=-\beta_{m}^{1}, & \gamma_{m}^{2}=-\gamma_{m}^{1}, \quad h_{0}=h_{1}=h_{2}=\alpha_{0}=\alpha_{1}=\alpha_{2}=0 .
\end{array}
$$

The bosons $\alpha_{m}^{1}, \beta_{m}^{1}, \gamma_{m}^{1}$ are the same bosons which were introduced to construct the elliptic current associated with the elliptic algebra $\mathrm{U}_{q, p}\left(\widehat{\mathfrak{s l}}_{2}\right)$ and the deformed Virasoro algebra $\operatorname{Vir}_{q, t}$ [7-9]. In order to construct infinitely many commutative operators, we introduce one parameter $s$ deformation of the bosons in [7-9]. This additional parameter $s$ plays an important role in proof of the main theorem.

We introduce the operators $C_{j}(z), C_{j}^{\dagger}(z), j=1,2$, acting on the Fock space $\mathcal{F}_{J, K}$

$$
\begin{aligned}
& C_{1}(z)=\mathrm{e}^{-\sqrt{\frac{2 r}{k(r-k)}} \mathrm{i} Q_{0}} \mathrm{e}^{-\sqrt{\frac{2 r}{k(r-k)}} P_{0} \log z}: \exp \left(-\sum_{m \neq 0} \alpha_{m}^{1} z^{-m}\right): \\
& C_{2}(z)=\mathrm{e}^{\sqrt{\frac{2 r}{k(r-k)}} \mathrm{i} Q_{0}} \mathrm{e}^{\sqrt{\frac{2 r}{k(r-k)}} P_{0} \log z}: \exp \left(-\sum_{m \neq 0} \alpha_{m}^{2} z^{-m}\right): \\
& C_{1}^{\dagger}(z)=\mathrm{e}^{\sqrt{\frac{2(r-k)}{k r}} \mathrm{i} Q_{0}} \mathrm{e}^{\sqrt{\frac{2(r-k)}{k r}} P_{0} \log z}: \exp \left(\sum_{m \neq 0} \widetilde{\alpha}_{m}^{1} z^{-m}\right): \\
& C_{2}^{\dagger}(z)=\mathrm{e}^{-\sqrt{\frac{2(r-k)}{k r}} \mathrm{i} Q_{0}} \mathrm{e}^{-\sqrt{\frac{2(r-k)}{k r}} P_{0} \log z}: \exp \left(\sum_{m \neq 0} \widetilde{\alpha}_{m}^{2} z^{-m}\right):
\end{aligned}
$$

Here : * : represents normal ordering. We set the operators $\widetilde{\Psi}_{j, I}(z), \widetilde{\Psi}_{j, I I}(z)$, $\widetilde{\Psi}_{j, I}^{\dagger}(z), \widetilde{\Psi}_{j, I I}^{\dagger}(z), j=1,2$, acting on the Fock space $\mathcal{F}_{J, K}$

$$
\begin{aligned}
\widetilde{\Psi}_{j, I}(z) & =\exp \left(-\left(x-x^{-1}\right) \sum_{m>0} \frac{x^{\frac{k m}{2}}}{[m]_{+}} \beta_{m}^{j} z^{-m}\right) \\
& \times \exp \left(-\sum_{m>0} x^{-\frac{k m}{2}} \gamma_{-m}^{j} z^{m}\right) \exp \left(-\sum_{m>0} x^{\frac{k m}{2}} \frac{[(k+1) m]_{+}}{[m]_{+}} \gamma_{m}^{j} z^{-m}\right)
\end{aligned}
$$




$$
\begin{aligned}
\widetilde{\Psi}_{j, I I}(z) & =\exp \left(\left(x-x^{-1}\right) \sum_{m>0} \frac{x^{\frac{k m}{2}}}{[m]_{+}} \beta_{-m}^{j} z^{m}\right) \\
& \times \exp \left(-\sum_{m>0} x^{\frac{k m}{2}} \frac{[(k+1) m]_{+}}{[m]_{+}} \gamma_{-m}^{j} z^{m}\right) \exp \left(-\sum_{m>0} x^{-\frac{k m}{2}} \gamma_{m}^{j} z^{-m}\right) \\
\widetilde{\Psi}_{j, I}^{\dagger}(z) & =\exp \left(\left(x-x^{-1}\right) \sum_{m>0} \frac{x^{-\frac{k m}{2}}}{[m]_{+}} \beta_{m}^{j} z^{-m}\right) \\
& \times \exp \left(\sum_{m>0} x^{\frac{k m}{2}} \gamma_{-m}^{j} z^{m}\right) \exp \left(\sum_{m>0} x^{-\frac{k m}{2}} \frac{[(k+1) m]_{+}}{[m]_{+}} \gamma_{m}^{j} z^{-m}\right) \\
\widetilde{\Psi}_{j, I I}^{\dagger}(z) & =\exp \left(-\left(x-x^{-1}\right) \sum_{m>0} \frac{x^{-\frac{k m}{2}}}{[m]_{+}} \beta_{-m}^{j} z^{m}\right) \\
& \times \exp \left(\sum_{m>0} x^{-\frac{k m}{2}} \frac{[(k+1) m]_{+}}{[m]_{+}} \gamma_{-m}^{j} z^{m}\right) \exp \left(\sum_{m>0} x^{\frac{k m}{2}} \gamma_{m}^{j} z^{-m}\right)
\end{aligned}
$$

We set the operators $\Psi_{j, I}(z), \Psi_{j, I I}(z), \Psi_{j, I}^{\dagger}(z), \Psi_{j, I I}^{\dagger}(z), j=1,2$, acting on the Fock space $\mathcal{F}_{J, K}$

$$
\begin{aligned}
\Psi_{1, I}(z) & =\widetilde{\Psi}_{1, I}(z) \mathrm{e}^{\alpha+\alpha_{0}+\alpha_{1}} x^{\frac{h}{2}+h_{0}+h_{1}} z^{-\frac{h}{k}} \\
\Psi_{1, I I}(z) & =\widetilde{\Psi}_{1, I I}(z) \mathrm{e}^{\alpha+\alpha_{0}+\alpha_{1}} x^{-\frac{h}{2}+h_{0}-h_{1}} z^{-\frac{h}{k}} \\
\Psi_{2, I}(z) & =\widetilde{\Psi}_{2, I}(z) \mathrm{e}^{-\alpha-\alpha_{0}+\alpha_{2}} x^{-\frac{h}{2}+h_{0}+h_{2}} z^{\frac{h}{k}} \\
\Psi_{2, I I}(z) & =\widetilde{\Psi}_{2, I I}(z) \mathrm{e}^{-\alpha-\alpha_{0}+\alpha_{2}} x^{\frac{h}{2}+h_{0}-h_{2}} z^{\frac{h}{k}} \\
\Psi_{1, I}^{\dagger}(z) & =\widetilde{\Psi}_{1, I}^{\dagger}(z) \mathrm{e}^{-\alpha-\alpha_{0}+\alpha_{1}} x^{\frac{h}{2}-h_{0}-h_{1}} z^{\frac{h}{k}} \\
\Psi_{1, I I}^{\dagger}(z) & =\widetilde{\Psi}_{1, I I}^{\dagger}(z) \mathrm{e}^{-\alpha-\alpha_{0}+\alpha_{1}} x^{-\frac{h}{2}-h_{0}+h_{1}} z^{\frac{h}{k}} \\
\Psi_{2, I}^{\dagger}(z) & =\widetilde{\Psi}_{2, I}^{\dagger}(z) \mathrm{e}^{\alpha+\alpha_{0}+\alpha_{2}} x^{-\frac{h}{2}-h_{0}-h_{2}} z^{-\frac{h}{k}} \\
\Psi_{2, I I}^{\dagger}(z) & =\widetilde{\Psi}_{2, I I}^{\dagger}(z) \mathrm{e}^{\alpha+\alpha_{0}+\alpha_{2}} x^{\frac{h}{2}-h_{0}+h_{2}} z^{-\frac{h}{k}} .
\end{aligned}
$$

Definition 1. We set the operators $E_{j}(z), F_{j}(z), j=1,2$, which can be regarded as one parameter deformation of the level $k$ elliptic currents associated with the elliptic algebra $\mathrm{U}_{q, p}\left(\widehat{\mathfrak{s l}_{2}}\right)[7,9]$

$$
E_{j}(z)=C_{j}(z) \Psi_{j}(z), \quad F_{j}(z)=C_{j}^{\dagger}(z) \Psi_{j}^{\dagger}(z), \quad j=1,2
$$


where we have set

$$
\begin{aligned}
& \Psi_{j}(z)=\frac{1}{x-x^{-1}}\left(\Psi_{j, I}(z)-\Psi_{j, I I}(z)\right) \\
& \Psi_{j}^{\dagger}(z)=\frac{-1}{x-x^{-1}}\left(\Psi_{j, I}^{\dagger}(z)-\Psi_{j, I I}^{\dagger}(z)\right), \quad j=1,2 .
\end{aligned}
$$

We have following as direct consequence of the normal orderings of the basic operators summarized in appendix.

Theorem 1. The elliptic currents $E_{j}(z), j=1,2$, satisfy the following commutation relations

$$
\begin{aligned}
& {\left[u_{1}-u_{2}\right]_{r-k}\left[u_{1}-u_{2}-1\right]_{r-k} E_{j}\left(z_{1}\right) E_{j}\left(z_{2}\right)} \\
& =\left[u_{2}-u_{1}\right]_{r-k}\left[u_{2}-u_{1}-1\right]_{r-k} E_{j}\left(z_{2}\right) E_{j}\left(z_{1}\right), \quad j=1,2 \\
& {\left[u_{1}-u_{2}+\frac{s}{2}\right]_{r-k}\left[u_{1}-u_{2}-\frac{s}{2}+1\right]_{r-k} E_{1}\left(z_{1}\right) E_{2}\left(z_{2}\right)} \\
& =\left[u_{2}-u_{1}+\frac{s}{2}\right]_{r-k}\left[u_{2}-u_{1}-\frac{s}{2}+1\right]_{r-k} E_{2}\left(z_{2}\right) E_{1}\left(z_{1}\right) .
\end{aligned}
$$

The elliptic currents $F_{j}(z), j=1,2$, satisfy the following commutation relations

$$
\begin{aligned}
& {\left[u_{1}-u_{2}\right]_{r} } {\left[u_{1}-u_{2}+1\right]_{r} F_{j}\left(z_{1}\right) F_{j}\left(z_{2}\right) } \\
&=\left[u_{2}-u_{1}\right]_{r}\left[u_{2}-u_{1}+1\right]_{r} F_{j}\left(z_{2}\right) F_{j}\left(z_{1}\right), \quad j=1,2 \\
& {\left[u_{1}-u_{2}-\frac{s}{2}\right]_{r}\left[u_{1}-u_{2}+\frac{s}{2}-1\right]_{r} F_{1}\left(z_{1}\right) F_{2}\left(z_{2}\right) } \\
& \quad=\left[u_{2}-u_{1}-\frac{s}{2}\right]_{r}\left[u_{2}-u_{1}+\frac{s}{2}-1\right]_{r} F_{2}\left(z_{2}\right) F_{1}\left(z_{1}\right) .
\end{aligned}
$$

The currents $E_{j}(z)$ and $F_{j}(z)$ satisfy

$$
\begin{gathered}
{\left[E_{j}\left(z_{1}\right), F_{j}\left(z_{2}\right)\right]=\frac{x^{(-1)^{j}(s-2)}}{x-x^{-1}}\left(: C_{j}\left(z_{1}\right) C_{j}^{\dagger}\left(z_{2}\right) \Psi_{j, I}\left(z_{1}\right) \Psi_{j, I}^{\dagger}\left(z_{2}\right): \delta\left(\frac{x^{k} z_{2}}{z_{1}}\right)\right.} \\
\left.-: C_{j}\left(z_{1}\right) C_{j}^{\dagger}\left(z_{2}\right) \Psi_{j, I I}\left(z_{1}\right) \Psi_{j, I I}^{\dagger}\left(z_{2}\right): \delta\left(\frac{x^{-k} z_{2}}{z_{1}}\right)\right), \quad j=1,2 .
\end{gathered}
$$

Here we have used the delta-function $\delta(z)=\sum_{n \in \mathbb{Z}} z^{n}$.

Upon specialization $s=2$ the currents $E_{1}(z), F_{1}(z)$ degenerate to elliptic currents in [9]. We set $E_{j}^{D V}(z)=\left.E_{j}(z)\right|_{s=2}, F_{j}^{D V}(z)=\left.F_{j}(z)\right|_{s=2}, j=1,2$. 


\section{Integrals of Motion}

In this section we construct infinitely many commutative operators $\mathcal{G}_{m}^{*}, \mathcal{G}_{m}, m \in$ $\mathbb{N}$, which we call the nonlocal integrals of motion for level $k$.

\subsection{Nonlocal Integrals of Motion}

Let us set the theta function $\vartheta_{\alpha}^{*}(u), \vartheta_{\alpha}(u), \alpha \in \mathbb{C}$, by

$$
\begin{aligned}
\vartheta^{*}(u+1) & =\vartheta^{*}(u) \\
\vartheta^{*}\left(u+r^{*} \tau^{*}\right) & =\exp \left[-2 \pi \sqrt{-1} \tau^{*}-\frac{2 \pi \sqrt{-1}}{r^{*}}\left(2 u-\sqrt{\frac{2 r r^{*}}{k}} P_{0}-\frac{r^{*}}{k} h\right)\right] \vartheta^{*}(u) \\
\vartheta(u+1) & =\vartheta(u) \\
\vartheta(u+r \tau) & =\exp \left[-2 \pi \sqrt{-1} \tau-\frac{2 \pi \sqrt{-1}}{r}\left(2 u-\sqrt{\frac{2 r r^{*}}{k}} P_{0}-\frac{r}{k} h\right)\right] \vartheta(u) .
\end{aligned}
$$

Let us use the parameterization $z_{j}^{(t)}=x^{2 u_{j}^{(t)}}, t=1,2, j=1,2, \ldots, m$.

Definition 2. We define the operator $\mathcal{G}_{m}^{*}$ for the regime $\operatorname{Re}(r)>k$ and $0<$ $\operatorname{Re}(s)<2$ by

$$
\begin{gathered}
\mathcal{G}_{m}^{*}=\int \cdots \int_{C^{*}} \prod_{j=1}^{m} \frac{\mathrm{d} z_{j}^{(1)}}{z_{j}^{(1)}} \prod_{j=1}^{m} \frac{\mathrm{d} z_{j}^{(2)}}{z_{j}^{(2)}} E_{1}\left(z_{1}^{(1)}\right) E_{1}\left(z_{2}^{(1)}\right) \cdots E_{1}\left(z_{m}^{(1)}\right) \\
\times E_{2}\left(z_{1}^{(2)}\right) E_{2}\left(z_{2}^{(2)}\right) \cdots E_{2}\left(z_{m}^{(2)}\right) \\
\left.\times \frac{\prod_{t=1,2} \prod_{1 \leqq i<j \leqq m}\left[u_{i}^{(t)}-u_{j}^{(t)}\right]_{r-k}\left[u_{j}^{(t)}-u_{i}^{(t)}+1\right]_{r-k}}{\prod_{1 \leqq i, j \leqq m}\left[u_{i}^{(1)}-u_{j}^{(2)}-\frac{s}{2}\right]_{r-k}\left[u_{j}^{(2)}-u_{i}^{(1)}-\frac{s}{2}+1\right]_{r-k}} \vartheta_{j=1}^{m}\left(u_{j}^{(2)}-u_{j}^{(1)}\right)\right)
\end{gathered}
$$

where the integral contour $C^{*}$ encircles $z_{j}^{(t)}=0, t=1,2, j=1,2, \ldots, m$, in such a way that

$$
\left|z_{j}^{(t)}\right|=1, \quad t=1,2, \quad j=1,2, \ldots, m .
$$

We define the operator $\mathcal{G}_{m}$ for the regime $\operatorname{Re}(r)>0$ and $0<\operatorname{Re}(s)<2$ by

$$
\mathcal{G}_{m}=\int \cdots \int_{C} \prod_{j=1}^{m} \frac{\mathrm{d} z_{j}^{(1)}}{z_{j}^{(1)}} \prod_{j=1}^{m} \frac{\mathrm{d} z_{j}^{(2)}}{z_{j}^{(2)}} F_{1}\left(z_{1}^{(1)}\right) F_{1}\left(z_{2}^{(1)}\right) \cdots F_{1}\left(z_{m}^{(1)}\right)
$$




$$
\begin{gathered}
\times F_{2}\left(z_{1}^{(2)}\right) F_{2}\left(z_{2}^{(2)}\right) \cdots F_{2}\left(z_{m}^{(2)}\right) \\
\times \frac{\prod_{t=1,2} \prod_{1 \leqq i<j \leqq m}\left[u_{i}^{(t)}-u_{j}^{(t)}\right]_{r}\left[u_{j}^{(t)}-u_{i}^{(t)}-1\right]_{r}}{\prod_{1 \leqq i, j \leqq m}\left[u_{i}^{(1)}-u_{j}^{(2)}+\frac{s}{2}\right]_{r}\left[u_{j}^{(2)}-u_{i}^{(1)}+\frac{s}{2}-1\right]_{r}} \vartheta\left(\sum_{j=1}^{m}\left(u_{j}^{(1)}-u_{j}^{(2)}\right)\right)
\end{gathered}
$$

where the integral contour $C^{*}$ encircles $z_{j}^{(t)}=0, t=1,2, j=1,2, \ldots, m$, in such a way that

$$
\left|z_{j}^{(t)}\right|=1, \quad t=1,2, \quad j=1,2, \ldots, m .
$$

We call the operators $\mathcal{G}_{m}^{*}$ and $\mathcal{G}_{m}$ the nonlocal integrals of motion for level $k$.

The definition of the operators $\mathcal{G}_{m}^{*}, \mathcal{G}_{m}$ for generic $s \in \mathbb{C}, s \neq 2$, should be understood as analytic continuation. In the limit $s \rightarrow 2$, the contour $C^{*}, C$ pinch at $z_{j}^{(t)}=z_{i}^{\left(t^{\prime}\right)}$. Hence the definition of $\mathcal{G}_{m}^{*}, \mathcal{G}_{m}$ do not hold for $s=2$. We give modified definition of $\mathcal{G}_{m}^{*}, \mathcal{G}_{m}$ for $s=2$, below. We note that parameter $s \neq 2$ plays an important role in the proof of Theorem 2 .

Definition 3. We define the operator $\mathcal{G}_{m}^{D V *}$ for the regime $\operatorname{Re}(r)>k$ and $s=2$ by

$$
\begin{gathered}
\mathcal{G}_{m}^{D V *}=\int \cdots \int_{C_{\mathrm{Arg}}^{*}} \prod_{j=1}^{m} \frac{\mathrm{d} z_{j}^{(1)}}{z_{j}^{(1)}} \prod_{j=1}^{m} \frac{\mathrm{d} z_{j}^{(2)}}{z_{j}^{(2)}} E_{1}^{D V}\left(z_{1}^{(1)}\right) \cdots E_{1}^{D V}\left(z_{m}^{(1)}\right) \\
\times E_{2}^{D V}\left(z_{1}^{(2)}\right) \cdots E_{2}^{D V}\left(z_{m}^{(2)}\right) \\
\left.\times \frac{\prod_{t=1,2} \prod_{1 \leqq i<j \leqq m}\left[u_{i}^{(t)}-u_{j}^{(t)}\right]_{r-k}\left[u_{j}^{(t)}-u_{i}^{(t)}+1\right]_{r-k}}{\prod_{1 \leqq i, j \leqq m}\left[u_{i}^{(1)}-u_{j}^{(2)}-1\right]_{r-k}\left[u_{j}^{(2)}-u_{i}^{(1)}\right]_{r-k}} \vartheta_{j=1}^{m}\left(u_{j}^{(2)}-u_{j}^{(1)}\right)\right)
\end{gathered}
$$

where the integral contour $C_{\mathrm{Arg}}^{*}$ encircles $z_{j}^{(t)}=0, t=1,2, j=1,2, \ldots, m$, in such a way that

$$
\left|x^{2} z_{m}^{(2)}\right|,\left|x^{2 r^{*}} z_{m}^{(2)}\right|<\left|z_{1}^{(1)}\right|<\left|z_{1}^{(2)}\right|<\left|z_{2}^{(1)}\right|<\left|z_{2}^{(2)}\right|<\cdots<\left|z_{m}^{(1)}\right|<\left|z_{m}^{(2)}\right| .
$$

We define the operator $\mathcal{G}_{m}^{D V}$ for the regime $\operatorname{Re}(r)>0$ and $s=2$ by

$$
\mathcal{G}_{m}^{D V}=\int \cdots \int_{C_{\mathrm{Arg}}} \prod_{j=1}^{m} \frac{\mathrm{d} z_{j}^{(1)}}{z_{j}^{(1)}} \prod_{j=1}^{m} \frac{\mathrm{d} z_{j}^{(2)}}{z_{j}^{(2)}} F_{1}^{D V}\left(z_{1}^{(1)}\right) \cdots F_{1}^{D V}\left(z_{m}^{(1)}\right)
$$




$$
\begin{gathered}
\times F_{2}^{D V}\left(z_{1}^{(2)}\right) \cdots F_{2}^{D V}\left(z_{m}^{(2)}\right) \\
\times \frac{\prod_{t=1,2} \prod_{1 \leqq i<j \leqq m}\left[u_{i}^{(t)}-u_{j}^{(t)}\right]_{r}\left[u_{j}^{(t)}-u_{i}^{(t)}-1\right]_{r}}{\prod_{1 \leqq i, j \leqq m}\left[u_{i}^{(1)}-u_{j}^{(2)}+1\right]_{r}\left[u_{j}^{(2)}-u_{i}^{(1)}\right]_{r}} \vartheta\left(\sum_{j=1}^{m}\left(u_{j}^{(1)}-u_{j}^{(2)}\right)\right)
\end{gathered}
$$

where the integral contour $C_{\mathrm{Arg}}$ encircles $z_{j}^{(t)}=0, t=1,2, j=1,2, \ldots, m$, in such a way that

$$
\left|x^{2} z_{m}^{(2)}\right|,\left|x^{2 r} z_{m}^{(2)}\right|<\left|z_{1}^{(1)}\right|<\left|z_{1}^{(2)}\right|<\left|z_{2}^{(1)}\right|<\left|z_{2}^{(2)}\right|<\cdots<\left|z_{m}^{(1)}\right|<\left|z_{m}^{(2)}\right| .
$$

\subsection{Main Result}

The following is the main theorem of this paper.

Theorem 2. For the regime $s \neq 2$ and $\operatorname{Re}(r)>k$ we have

$$
\left[\mathcal{G}_{m}^{*}, \mathcal{G}_{n}^{*}\right]=0, \quad m, n \in \mathbb{N} .
$$

For the regime $s \neq 2$ and $\operatorname{Re}(r)>0$ we have

$$
\left[\mathcal{G}_{m}, \mathcal{G}_{n}\right]=0, \quad m, n \in \mathbb{N} \text {. }
$$

Let us sketch the proof of Theorem 2. The proof is given as the same manner as level $k=1$ case in $[1,3]$. By symmetrization of the screenings $E_{j}(z)$ the commutation relation $\left[\mathcal{G}_{m}^{*}, \mathcal{G}_{n}^{*}\right]=0$ is reduced to the following sufficient condition of the theta functions, which is shown by induction as the same manner as $[1,3]$. We note that this symmetrization procedure holds only for $s \neq 2$

$$
\begin{aligned}
& \sum_{\substack{K \cup K^{c}=L \cup L^{c}=\{1,2, \ldots, n+m\} \\
|K|=|L|=n,\left|K^{c}\right|=\left|L^{c}\right|=m}} \vartheta^{*}\left(\sum_{j \in K^{c}} u_{j}^{(2)}-\sum_{j \in L^{c}} u_{j}^{(1)}\right) \vartheta^{*}\left(\sum_{j \in K} u_{j}^{(2)}-\sum_{j \in L} u_{j}^{(1)}\right) \\
\times & \prod_{\substack{i \in K^{c} \\
p \in K^{c}}} \prod_{\substack{j \in K^{c} \\
q \in K^{c}}} \frac{\left[u_{j}^{(2)}-u_{p}^{(1)}-\frac{s}{2}\right]_{r-k}\left[u_{i}^{(1)}-u_{q}^{(2)}-\frac{s}{2}\right]_{r-k}}{\left[u_{i}^{(1)}-u_{p}^{(1)}\right]_{r-k}\left[u_{j}^{(2)}-u_{q}^{(2)}\right]_{r-k}} \\
\times & \prod_{\substack{i \in K^{c} \\
p \in K^{c}}} \prod_{\substack{j \in K^{c} \\
q \in K^{c}}} \frac{\left[u_{p}^{(1)}-u_{j}^{(2)}-\frac{s}{2}+1\right]_{r-k}\left[u_{q}^{(2)}-u_{i}^{(1)}-\frac{s}{2}+1\right]_{r-k}}{\left[u_{p}^{(1)}-u_{i}^{(1)}+1\right]_{r-k}\left[u_{q}^{(2)}-u_{j}^{(2)}+1\right]_{r-k}}
\end{aligned}
$$




$$
\begin{aligned}
& =\sum_{\substack{K \cup K^{c}=L \cup L^{c}=\{1,2, \ldots, n+m\} \\
|K|=|L|=n,\left|K^{c}\right|=\left|L^{c}\right|=m}} \vartheta^{*}\left(\sum_{j \in K^{c}} u_{j}^{(2)}-\sum_{j \in L^{c}} u_{j}^{(1)}\right) \vartheta^{*}\left(\sum_{j \in K} u_{j}^{(2)}-\sum_{j \in L} u_{j}^{(1)}\right) \\
& \times \prod_{\substack{i \in K^{c} \\
p \in K^{c}}} \prod_{\substack{j \in K^{c} \\
q \in K^{c}}} \frac{\left[u_{q}^{(2)}-u_{i}^{(1)}-\frac{s}{2}\right]_{r-k}\left[u_{p}^{(2)}-u_{j}^{(1)}-\frac{s}{2}\right]_{r-k}}{\left[u_{p}^{(1)}-u_{i}^{(1)}\right]_{r-k}\left[u_{q}^{(2)}-u_{j}^{(2)}\right]_{r-k}} \\
& \times \prod_{\substack{i \in K^{c} \\
p \in K^{c}}} \prod_{\substack{j \in K^{c} \\
q \in K^{c}}} \frac{\left[u_{i}^{(1)}-u_{q}^{(2)}-\frac{s}{2}+1\right]_{r-k}\left[u_{j}^{(2)}-u_{p}^{(1)}-\frac{s}{2}+1\right]_{r-k}}{\left[u_{i}^{(1)}-u_{p}^{(1)}+1\right]_{r-k}\left[u_{q}^{(2)}-u_{j}^{(2)}+1\right]_{r-k}}
\end{aligned}
$$

Naively, when we take the limit $s \rightarrow 2$, it seems that we have $\left[\mathcal{G}_{m}^{D V *}, \mathcal{G}_{n}^{D V *}\right]=0$. However, very precisely, in order to take the limit $s \rightarrow 2$, we have to consider special treatment which we call "renormalized" limit in [1]. Here we state only conjecture on the operator $\mathcal{G}_{m}^{D V *}$. Theorem 2 give a supporting argument of the following conjecture.

Conjecture 3. For the regime $s=2$ and $\operatorname{Re}(r)>k$ we have

$$
\left[\mathcal{G}_{m}^{D V *}, \mathcal{G}_{n}^{D V *}\right]=0, \quad m, n \in \mathbb{N} .
$$

For the regime $s=2$ and $\operatorname{Re}(r)>0$ we have

$$
\left[\mathcal{G}_{m}^{D V}, \mathcal{G}_{n}^{D V}\right]=0, \quad m, n \in \mathbb{N} .
$$

In this paper we gave one parameter " $s$ " deformation of level $k$ free field realization of the screening current of the elliptic algebra $\mathrm{U}_{q, p}\left(\widehat{\mathfrak{s l}_{2}}\right)$. By means of these free field realizations, we constructed infinitely many commutative operators, which we call the nonlocal integrals of motion associated with the elliptic algebra $\mathrm{U}_{q, p}\left(\widehat{\mathfrak{s l}_{2}}\right)$ for arbitrary level $k \neq 0,-2$. They are given as integrals involving a product of the screening current and Jacobi elliptic theta functions. The construction of the local integrals of motion $\mathcal{I}_{m}$ for arbitrary level $k$ is open problem. The construction of the local integrals of motion $\mathcal{I}_{m}$ for level 1 only is summarized in [1-3].

\section{Acknowledgements}

We would like to thank the organizing committee of the Tenth International Conference on Geometry, Integrability and Quantization held at Sts. Constantine and 
Elena, Bulgaria. We would like to thank Professors V. Bazhanov, P. Bouwknegt, A. Chervov, V. Gerdjikov, F. Goehmann, K. Hasegawa, M. Jimbo, A. Kluemper, P. Kulish, W.-X. Ma, V. Mangazeev and I. Mladenov for their interest in this work. This work is partly supported by Grant-in Aid for Young Scientist B (18740092) from JSPS.

\section{Appendix}

Here we summarize the normal orderings of the basic operators (in all cases the subscript $j=1,2$ ).

$$
\begin{aligned}
& C_{j}\left(z_{1}\right) C_{j}\left(z_{2}\right)=:: z_{1}^{\frac{2}{r^{*}}+\frac{2}{k}} \frac{\left(x^{-2+2 k} z_{2} / z_{1} ; x^{2 r^{*}}\right)_{\infty}\left(x^{-2} z_{2} / z_{1} ; x^{2 k}\right)_{\infty}}{\left(x^{2+2 k} z_{2} / z_{1} ; x^{2 r^{*}}\right)_{\infty}\left(x^{2} z_{2} / z_{1} ; x^{2 k}\right)_{\infty}} \\
& C_{1}\left(z_{1}\right) C_{2}\left(z_{2}\right)=:: z_{1}^{-\frac{2}{r^{*}}-\frac{2}{k}} \frac{\left(x^{s} z_{2} / z_{1} ; x^{2 r^{*}}\right)_{\infty}\left(x^{2-s} z_{2} / z_{1} ; x^{2 r^{*}}\right)_{\infty}}{\left(x^{-s} z_{2} / z_{1} ; x^{2 r^{*}}\right)_{\infty}\left(x^{s-2} z_{2} / z_{1} ; x^{2 r^{*}}\right)_{\infty}} \\
& \times \frac{\left(x^{s+2 k} z_{2} / z_{1} ; x^{2 k}\right)_{\infty}\left(x^{s-2+2 k} z_{2} / z_{1} ; x^{2 k}\right)_{\infty}}{\left(x^{-s+2 k} z_{2} / z_{1} ; x^{2 k}\right)_{\infty}\left(x^{2-s+2 k} z_{2} / z_{1} ; x^{2 k}\right)_{\infty}} \\
& C_{2}\left(z_{1}\right) C_{1}\left(z_{2}\right)=:: z_{1}^{-\frac{2}{r^{*}}-\frac{2}{k}} \frac{\left(x^{s+2 r^{*}} z_{2} / z_{1} ; x^{2 r^{*}}\right)_{\infty}\left(x^{2-s+2 r^{*}} z_{2} / z_{1} ; x^{2 r^{*}}\right)_{\infty}}{\left(x^{-s+2 r^{*}} z_{2} / z_{1} ; x^{2 r^{*}}\right)_{\infty}\left(x^{s-2+2 r^{*}} z_{2} / z_{1} ; x^{2 r^{*}}\right)_{\infty}} \\
& \times \frac{\left(x^{s} z_{2} / z_{1} ; x^{2 k}\right)_{\infty}\left(x^{s-2} z_{2} / z_{1} ; x^{2 k}\right)_{\infty}}{\left(x^{-s} z_{2} / z_{1} ; x^{2 k}\right)_{\infty}\left(x^{2-s} z_{2} / z_{1} ; x^{2 k}\right)_{\infty}} \\
& C_{j}^{\dagger}\left(z_{1}\right) C_{j}^{\dagger}\left(z_{2}\right)=:: z_{1}^{-\frac{2}{r}+\frac{2}{k}} \frac{\left(x^{-2+2 k} z_{1} / z_{2} ; x^{2 k}\right)_{\infty}\left(x^{2+2 r} z_{1} / z_{2} ; x^{2 r}\right)_{\infty}}{\left(x^{2+2 k} z_{2} / z_{1} ; x^{2 k}\right)_{\infty}\left(x^{-2+2 r} z_{2} / z_{1} ; x^{2 r}\right)_{\infty}} \\
& C_{1}^{\dagger}\left(z_{1}\right) C_{2}^{\dagger}\left(z_{2}\right)=:: z_{1}^{\frac{2}{r}-\frac{2}{k}} \frac{\left(x^{s+2 k} z_{2} / z_{1} ; x^{2 k}\right)_{\infty}\left(x^{s-2+2 k} z_{2} / z_{1} ; x^{2 k}\right)_{\infty}}{\left(x^{-s+2 k} z_{2} / z_{1} ; x^{2 k}\right)_{\infty}\left(x^{2-s+2 k} z_{2} / z_{1} ; x^{2 k}\right)_{\infty}} \\
& \times \frac{\left(x^{-s+2 r} z_{2} / z_{1} ; x^{2 r}\right)_{\infty}\left(x^{s-2+2 r} z_{2} / z_{1} ; x^{2 r}\right)_{\infty}}{\left(x^{s+2 r} z_{2} / z_{1} ; x^{2 r}\right)_{\infty}\left(x^{2-s+2 r} z_{2} / z_{1} ; x^{2 r}\right)_{\infty}} \\
& C_{2}^{\dagger}\left(z_{1}\right) C_{1}^{\dagger}\left(z_{2}\right)=:: z_{1}^{\frac{2}{r}-\frac{2}{k}} \frac{\left(x^{s} z_{2} / z_{1} ; x^{2 k}\right)_{\infty}\left(x^{s-2} z_{2} / z_{1} ; x^{2 k}\right)_{\infty}}{\left(x^{-s} z_{2} / z_{1} ; x^{2 k}\right)_{\infty}\left(x^{2-s} z_{2} / z_{1} ; x^{2 k}\right)_{\infty}} \\
& \times \frac{\left(x^{-s} z_{2} / z_{1} ; x^{2 r}\right)_{\infty}\left(x^{s-2} z_{2} / z_{1} ; x^{2 r}\right)_{\infty}}{\left(x^{s} z_{2} / z_{1} ; x^{2 r}\right)_{\infty}\left(x^{2-s} z_{2} / z_{1} ; x^{2 r}\right)_{\infty}} \\
& C_{j}\left(z_{1}\right) C_{j}^{\dagger}\left(z_{2}\right)=:: z_{1}^{-\frac{2}{k}} \frac{\left(x^{2+k} z_{2} / z_{1} ; x^{2 k}\right)_{\infty}}{\left(x^{-2+k} z_{2} / z_{1} ; x^{2 k}\right)_{\infty}} \\
& C_{j}^{\dagger}\left(z_{1}\right) C_{j}\left(z_{2}\right)=:: z_{1}^{-\frac{2}{k}} \frac{\left(x^{2+k} z_{2} / z_{1} ; x^{2 k}\right)_{\infty}}{\left(x^{-2+k} z_{2} / z_{1} ; x^{2 k}\right)_{\infty}}
\end{aligned}
$$




$$
\begin{aligned}
& \widetilde{\Psi}_{1, I}\left(z_{1}\right) \widetilde{\Psi}_{2, I}\left(z_{2}\right)=:: \frac{\left(x^{-s} z_{2} / z_{1} ; x^{2 k}\right)_{\infty}\left(x^{2-s+2 k} z_{2} / z_{1} ; x^{2 k}\right)_{\infty}}{\left(x^{s+2 k} z_{2} / z_{1} ; x^{2 k}\right)_{\infty}\left(x^{s-2} z_{2} / z_{1} ; x^{2 k}\right)_{\infty}} \\
& \widetilde{\Psi}_{2, I}\left(z_{1}\right) \widetilde{\Psi}_{1, I}\left(z_{2}\right)=:: \frac{\left(x^{-s} z_{2} / z_{1} ; x^{2 k}\right)_{\infty}\left(x^{2-s+2 k} z_{2} / z_{1} ; x^{2 k}\right)_{\infty}}{\left(x^{s+2 k} z_{2} / z_{1} ; x^{2 k}\right)_{\infty}\left(x^{s-2} z_{2} / z_{1} ; x^{2 k}\right)_{\infty}} \\
& \widetilde{\Psi}_{1, I I}\left(z_{1}\right) \widetilde{\Psi}_{2, I I}\left(z_{2}\right)=:: \frac{\left(x^{-s} z_{2} / z_{1} ; x^{2 k}\right)_{\infty}\left(x^{2-s+2 k} z_{2} / z_{1} ; x^{2 k}\right)_{\infty}}{\left(x^{s+2 k} z_{2} / z_{1} ; x^{2 k}\right)_{\infty}\left(x^{s-2} z_{2} / z_{1} ; x^{2 k}\right)_{\infty}} \\
& \widetilde{\Psi}_{2, I I}\left(z_{1}\right) \widetilde{\Psi}_{1, I I}\left(z_{2}\right)=:: \frac{\left(x^{-s} z_{2} / z_{1} ; x^{2 k}\right)_{\infty}\left(x^{2-s+2 k} z_{2} / z_{1} ; x^{2 k}\right)_{\infty}}{\left(x^{s+2 k} z_{2} / z_{1} ; x^{2 k}\right)_{\infty}\left(x^{s-2} z_{2} / z_{1} ; x^{2 k}\right)_{\infty}} \\
& \widetilde{\Psi}_{1, I}^{\dagger}\left(z_{1}\right) \widetilde{\Psi}_{2, I}^{\dagger}\left(z_{2}\right)=:: \frac{\left(x^{-s} z_{2} / z_{1} ; x^{2 k}\right)_{\infty}\left(x^{2-s+2 k} z_{2} / z_{1} ; x^{2 k}\right)_{\infty}}{\left(x^{s+2 k} z_{2} / z_{1} ; x^{2 k}\right)_{\infty}\left(x^{s-2} z_{2} / z_{1} ; x^{2 k}\right)_{\infty}} \\
& \widetilde{\Psi}_{2, I}^{\dagger}\left(z_{1}\right) \widetilde{\Psi}_{1, I}^{\dagger}\left(z_{2}\right)=:: \frac{\left(x^{-s} z_{2} / z_{1} ; x^{2 k}\right)_{\infty}\left(x^{2-s+2 k} z_{2} / z_{1} ; x^{2 k}\right)_{\infty}}{\left(x^{s+2 k} z_{2} / z_{1} ; x^{2 k}\right)_{\infty}\left(x^{s-2} z_{2} / z_{1} ; x^{2 k}\right)_{\infty}} \\
& \widetilde{\Psi}_{1, I I}^{\dagger}\left(z_{1}\right) \widetilde{\Psi}_{2, I I}^{\dagger}\left(z_{2}\right)=:: \frac{\left(x^{-s} z_{2} / z_{1} ; x^{2 k}\right)_{\infty}\left(x^{2-s+2 k} z_{2} / z_{1} ; x^{2 k}\right)_{\infty}}{\left(x^{s+2 k} z_{2} / z_{1} ; x^{2 k}\right)_{\infty}\left(x^{s-2} z_{2} / z_{1} ; x^{2 k}\right)_{\infty}} \\
& \widetilde{\Psi}_{2, I I}^{\dagger}\left(z_{1}\right) \widetilde{\Psi}_{1, I I}^{\dagger}\left(z_{2}\right)=:: \frac{\left(x^{-s} z_{2} / z_{1} ; x^{2 k}\right)_{\infty}\left(x^{2-s+2 k} z_{2} / z_{1} ; x^{2 k}\right)_{\infty}}{\left(x^{s+2 k} z_{2} / z_{1} ; x^{2 k}\right)_{\infty}\left(x^{s-2} z_{2} / z_{1} ; x^{2 k}\right)_{\infty}} \\
& \widetilde{\Psi}_{1, I}\left(z_{1}\right) \widetilde{\Psi}_{2, I I}\left(z_{2}\right)=:: \frac{\left(x^{-s+2 k} z_{2} / z_{1} ; x^{2 k}\right)_{\infty}\left(x^{2-s+2 k} z_{2} / z_{1} ; x^{2 k}\right)_{\infty}}{\left(x^{s+2 k} z_{2} / z_{1} ; x^{2 k}\right)_{\infty}\left(x^{s-2+2 k} z_{2} / z_{1} ; x^{2 k}\right)_{\infty}} \\
& \widetilde{\Psi}_{2, I I}\left(z_{1}\right) \widetilde{\Psi}_{1, I}\left(z_{2}\right)=:: \frac{\left(x^{-s} z_{2} / z_{1} ; x^{2 k}\right)_{\infty}\left(x^{2-s} z_{2} / z_{1} ; x^{2 k}\right)_{\infty}}{\left(x^{s} z_{2} / z_{1} ; x^{2 k}\right)_{\infty}\left(x^{s-2} z_{2} / z_{1} ; x^{2 k}\right)_{\infty}} \\
& \widetilde{\Psi}_{1, I I}\left(z_{1}\right) \widetilde{\Psi}_{2, I}\left(z_{2}\right)=:: \frac{\left(x^{-s} z_{2} / z_{1} ; x^{2 k}\right)_{\infty}\left(x^{2-s} z_{2} / z_{1} ; x^{2 k}\right)_{\infty}}{\left(x^{s} z_{2} / z_{1} ; x^{2 k}\right)_{\infty}\left(x^{s-2} z_{2} / z_{1} ; x^{2 k}\right)_{\infty}} \\
& \widetilde{\Psi}_{2, I}\left(z_{1}\right) \widetilde{\Psi}_{1, I I}\left(z_{2}\right)=:: \frac{\left(x^{-s+2 k} z_{2} / z_{1} ; x^{2 k}\right)_{\infty}\left(x^{2-s+2 k} z_{2} / z_{1} ; x^{2 k}\right)_{\infty}}{\left(x^{s+2 k} z_{2} / z_{1} ; x^{2 k}\right)_{\infty}\left(x^{s-2+2 k} z_{2} / z_{1} ; x^{2 k}\right)_{\infty}} \\
& \widetilde{\Psi}_{1, I}^{\dagger}\left(z_{1}\right) \widetilde{\Psi}_{2, I I}^{\dagger}\left(z_{2}\right)=:: \frac{\left(x^{-s} z_{2} / z_{1} ; x^{2 k}\right)_{\infty}\left(x^{2-s} z_{2} / z_{1} ; x^{2 k}\right)_{\infty}}{\left(x^{s} z_{2} / z_{1} ; x^{2 k}\right)_{\infty}\left(x^{s-2} z_{2} / z_{1} ; x^{2 k}\right)_{\infty}} \\
& \widetilde{\Psi}_{2, I I}^{\dagger}\left(z_{1}\right) \widetilde{\Psi}_{1, I}^{\dagger}\left(z_{2}\right)=:: \frac{\left(x^{-s+2 k} z_{2} / z_{1} ; x^{2 k}\right)_{\infty}\left(x^{2-s+2 k} z_{2} / z_{1} ; x^{2 k}\right)_{\infty}}{\left(x^{s+2 k} z_{2} / z_{1} ; x^{2 k}\right)_{\infty}\left(x^{s-2+2 k} z_{2} / z_{1} ; x^{2 k}\right)_{\infty}} \\
& \widetilde{\Psi}_{1, I I}^{\dagger}\left(z_{1}\right) \widetilde{\Psi}_{2, I}^{\dagger}\left(z_{2}\right)=:: \frac{\left(x^{-s+2 k} z_{2} / z_{1} ; x^{2 k}\right)_{\infty}\left(x^{2-s+2 k} z_{2} / z_{1} ; x^{2 k}\right)_{\infty}}{\left(x^{s+2 k} z_{2} / z_{1} ; x^{2 k}\right)_{\infty}\left(x^{s-2+2 k} z_{2} / z_{1} ; x^{2 k}\right)_{\infty}} \\
& \widetilde{\Psi}_{2, I}^{\dagger}\left(z_{1}\right) \widetilde{\Psi}_{1, I I}^{\dagger}\left(z_{2}\right)=:: \frac{\left(x^{-s} z_{2} / z_{1} ; x^{2 k}\right)_{\infty}\left(x^{2-s} z_{2} / z_{1} ; x^{2 k}\right)_{\infty}}{\left(x^{s} z_{2} / z_{1} ; x^{2 k}\right)_{\infty}\left(x^{s-2} z_{2} / z_{1} ; x^{2 k}\right)_{\infty}} \\
& \widetilde{\Psi}_{j, I}\left(z_{1}\right) \widetilde{\Psi}_{j, I}\left(z_{2}\right)=::\left(1-z_{2} / z_{1}\right) \frac{\left(x^{2+2 k} z_{2} / z_{1} ; x^{2 k}\right)_{\infty}}{\left(x^{-2} z_{2} / z_{1} ; x^{2 k}\right)_{\infty}}
\end{aligned}
$$




$$
\begin{aligned}
& \widetilde{\Psi}_{j, I I}\left(z_{1}\right) \widetilde{\Psi}_{j, I I}\left(z_{2}\right)=::\left(1-z_{2} / z_{1}\right) \frac{\left(x^{2+2 k} z_{2} / z_{1} ; x^{2 k}\right)_{\infty}}{\left(x^{-2} z_{2} / z_{1} ; x^{2 k}\right)_{\infty}} \\
& \widetilde{\Psi}_{j, I}\left(z_{1}\right) \widetilde{\Psi}_{j, I I}\left(z_{2}\right)=:: \frac{\left(x^{2+2 k} z_{2} / z_{1} ; x^{2 k}\right)_{\infty}}{\left(x^{-2+2 k} z_{2} / z_{1} ; x^{2 k}\right)_{\infty}} \\
& \widetilde{\Psi}_{j, I I}\left(z_{1}\right) \widetilde{\Psi}_{j, I}\left(z_{2}\right)=:: \frac{\left(x^{2} z_{2} / z_{1} ; x^{2 k}\right)_{\infty}}{\left(x^{-2} z_{2} / z_{1} ; x^{2 k}\right)_{\infty}} \\
& \widetilde{\Psi}_{j, I}^{\dagger}\left(z_{1}\right) \widetilde{\Psi}_{j, I}^{\dagger}\left(z_{2}\right)=::\left(1-z_{2} / z_{1}\right) \frac{\left(x^{2+2 k} z_{2} / z_{1} ; x^{2 k}\right)_{\infty}}{\left(x^{-2} z_{2} / z_{1} ; x^{2 k}\right)_{\infty}} \\
& \widetilde{\Psi}_{j, I I}^{\dagger}\left(z_{1}\right) \widetilde{\Psi}_{j, I I}^{\dagger}\left(z_{2}\right)=::\left(1-z_{2} / z_{1}\right) \frac{\left(x^{2+2 k} z_{2} / z_{1} ; x^{2 k}\right)_{\infty}}{\left(x^{-2} z_{2} / z_{1} ; x^{2 k}\right)_{\infty}} \\
& \widetilde{\Psi}_{j, I}^{\dagger}\left(z_{1}\right) \widetilde{\Psi}_{j, I I}^{\dagger}\left(z_{2}\right)=:: \frac{\left(x^{2} z_{2} / z_{1} ; x^{2 k}\right)_{\infty}}{\left(x^{-2} z_{2} / z_{1} ; x^{2 k}\right)_{\infty}} \\
& \widetilde{\Psi}_{j, I I}^{\dagger}\left(z_{1}\right) \widetilde{\Psi}_{j, I}^{\dagger}\left(z_{2}\right)=:: \frac{\left(x^{2+2 k} z_{2} / z_{1} ; x^{2 k}\right)_{\infty}}{\left(x^{-2+2 k} z_{2} / z_{1} ; x^{2 k}\right)_{\infty}} \\
& \widetilde{\Psi}_{j, I}\left(z_{1}\right) \widetilde{\Psi}_{j, I}^{\dagger}\left(z_{2}\right)=:: \frac{1}{\left(1-x^{k} z_{2} / z_{1}\right)} \frac{\left(x^{k-2} z_{2} / z_{1} ; x^{2 k}\right)_{\infty}}{\left(x^{3 k+2} z_{2} / z_{1} ; x^{2 k}\right)_{\infty}} \\
& \widetilde{\Psi}_{j, I}^{\dagger}\left(z_{1}\right) \widetilde{\Psi}_{j, I}\left(z_{2}\right)=:: \frac{1}{\left(1-x^{-k} z_{2} / z_{1}\right)} \frac{\left(x^{-k-2} z_{2} / z_{1} ; x^{2 k}\right)_{\infty}}{\left(x^{k+2} z_{2} / z_{1} ; x^{2 k}\right)_{\infty}} \\
& \widetilde{\Psi}_{j, I}\left(z_{1}\right) \widetilde{\Psi}_{j, I I}^{\dagger}\left(z_{2}\right)=:: \frac{\left(x^{-2+k} z_{2} / z_{1} ; x^{2 k}\right)_{\infty}}{\left(x^{2+k} z_{2} / z_{1} ; x^{2 k}\right)_{\infty}} \\
& \widetilde{\Psi}_{j, I I}^{\dagger}\left(z_{1}\right) \widetilde{\Psi}_{j, I}\left(z_{2}\right)=:: \frac{\left(x^{-2+k} z_{2} / z_{1} ; x^{2 k}\right)_{\infty}}{\left(x^{2+k} z_{2} / z_{1} ; x^{2 k}\right)_{\infty}} \\
& \widetilde{\Psi}_{j, I I}\left(z_{1}\right) \widetilde{\Psi}_{j, I}^{\dagger}\left(z_{2}\right)=:: \frac{\left(x^{-2+k} z_{2} / z_{1} ; x^{2 k}\right)_{\infty}}{\left(x^{2+k} z_{2} / z_{1} ; x^{2 k}\right)_{\infty}} \\
& \widetilde{\Psi}_{j, I}^{\dagger}\left(z_{1}\right) \widetilde{\Psi}_{j, I I}\left(z_{2}\right)=:: \frac{\left(x^{-2+k} z_{2} / z_{1} ; x^{2 k}\right)_{\infty}}{\left(x^{2+k} z_{2} / z_{1} ; x^{2 k}\right)_{\infty}} \\
& \widetilde{\Psi}_{j, I I}\left(z_{1}\right) \widetilde{\Psi}_{j, I I}^{\dagger}\left(z_{2}\right)=:: \frac{1}{\left(1-x^{k} z_{2} / z_{1}\right)} \frac{\left(x^{-k-2} z_{2} / z_{1} ; x^{2 k}\right)_{\infty}}{\left(x^{k+2} z_{2} / z_{1} ; x^{2 k}\right)_{\infty}} \\
& \widetilde{\Psi}_{j, I I}^{\dagger}\left(z_{1}\right) \widetilde{\Psi}_{j, I I}\left(z_{2}\right)=:: \frac{1}{\left(1-x^{k} z_{2} / z_{1}\right)} \frac{\left(x^{k-2} z_{2} / z_{1} ; x^{2 k}\right)_{\infty}}{\left(x^{3 k+2} z_{2} / z_{1} ; x^{2 k}\right)_{\infty}} .
\end{aligned}
$$




\section{References}

[1] Feigin B., Kojima T., Shiraishi J. and Watanabe H., The Integrals of Motion for the Deformed Virasoro Algebra, Preprint 2007, 54 pp (submitted for publication, arXiv:0705.0427).

[2] Feigin B., Kojima T., Shiraishi J. and Watanabe H., The Integrals of Motion for the Deformed $W$-algebra $W_{q, t}\left(\widehat{\mathfrak{s l}_{N}}\right)$, In: Proc. Representation Theory, 2006 (Atami, Japan), (2006) pp 102-114, ISBN 4-9902328-2-8.

[3] Kojima T. and Shiraishi J., The Integrals of Motion for the Deformed $W$ Algebra $W_{q, t}\left(\widehat{\mathfrak{g l}_{N}}\right)$ II: Proof of the Commutation Relations, Commun. Math. Phys. 283 (2008) 795-851.

[4] Bazhanov V., Lukyanov S. and Zamolodchikov Al., Integrable Structure of Conformal Field Theory: Quantum KdV Theory and Thermodynamic Bethe Ansatz, Commun. Math. Phys. 177 (1996) 381-398.

[5] Bazhanov V., Hibberd A. and Khoroshkin S., Integrable Structure of $W_{3}$ Conformal Field Theory, Quantum Boussinesq Theory and Boundary Affine Toda Theory, Nucl. Phys. B 622 (2002) 475-547.

[6] Kojima T., Baxter's Q-Operator for the $W$-Algebra $W_{N}$, J. Phys. A: Math. \& Theor. 41 (2008) 355206 (16 pp).

[7] Konno H., An Elliptic Algebra $\mathrm{U}_{q, p}\left(\widehat{\mathfrak{s t}_{2}}\right)$ and the Fusion RSOS Model, Commun. Math. Phys. 195 (1998) 373-403.

[8] Matsuo A., A q-Deformation of Wakimoto Modules, Primary Fields and Screening Operators, Commun. Math. Phys. 161 (1994) 33-48.

[9] Jimbo M., Konno H., Odake S. and Shiraishi J., Elliptic Algebra $\mathrm{U}_{q, p}\left(\widehat{\mathfrak{s l}_{2}}\right)$ : Drinfeld Current and Vertex Operators, Commun. Math. Phys. 199 (1999) 605-647.

[10] Jimbo M., Konno H., Odake S. and Shiraishi J., Quasi-Hopf Twistors for Elliptic Quantum Groups, Transformation Group 4 (1999) 303-327.

[11] Feigin B. and Odesskii A., A Family of Elliptic Algebras, Internat. Math. Res. Notices 11 (1997) 531-539.

Takeo Kojima

Department of Mathematics

College of Science and Technology

Nihon University

Surugadai, Chiyoda-ku

Tokyo 101-0062

JAPAN

E-mail address: kojima@math.cst.nihon_u.ac.jp 
Jun'ichi Shiraishi

Graduate School of Mathematical Science

University of Tokyo

Komaba, Megro-ku

Tokyo, 153-8914

JAPAN 